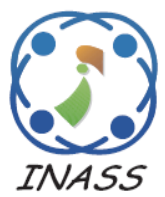

\title{
Genetic Algorithm Based Feature Selection Brain Tumour Segmentation and Classification
}

\author{
Vemula Vinay Kumar ${ }^{1 *}$ \\ Kodali Sai Krishna ${ }^{1}$ \\ Sarukonda Kusumavathi ${ }^{1}$ \\ ${ }^{1}$ Department Electronics and Communication, Anurag Group of Institutions, Hyderabad, India \\ * Corresponding author's Email: vemulavinay387@gmail.com
}

\begin{abstract}
Brain tumour segmentation is a significant task in the Medical Image Processing (MIP). Early diagnosis of the brain tumours plays an important role in improving treatment possibilities and maximizes the survival rate of the patients. Manual segmentation of the brain tumours for the cancer diagnosis form large amount of MRI images that generated in clinical routine, its complex and time consuming task. So, the automatic brain tumour image segmentation process is required. In this research, a tumour portion is segmented from the brain image by using Adaptively Regularized Kernel-Based Fuzzy C-Means (ARKFCM) algorithm. The input images are resized like 256×256 in the pre - processing stage. The pre-processed MRI image segmented by ARKFCM, which is a flexible high-level Machine Learning (ML) technique to locate the object in a complex template. Next, Hybrid Feature Extraction (HFE) performed on the segmented image to improve the feature subsets. The feature selection process was performed by Kernel Nearest Neighbour (KNN) based Genetic Algorithm (GA) in order to acquire the best feature values. The best feature values given to the Deep Neural Network (DNN) classifier as an input, which is classified into Meningioma, Glioma and Pituitary regions in the MRI images. The performance of proposed ARKFCM - HFE - DNN method is validated by T1-WCEMRI dataset. The experimental outcome showed that the ARKFCM - HFE - DNN method improved the average classification accuracy up to $6.19 \%$ than existing classification techniques such as Convolution Neural Network (CNN) and Support Vector Machine (SVM).
\end{abstract}

Keywords: Adaptively regularized kernel-based fuzzy c-means, Deep neural network, Genetic algorithm, Hybrid feature extraction, Kernel nearest neighbour.

\section{Introduction}

Image segmentation defines the process of dividing a digital image from multiple regions of the image. The major goal of the segmentation process is to estimate the location of boundaries and objects from images $[1,2]$. Generally, the anatomy of the brain tumor can be scanned by Magnitude Resonance Imaging (MRI) scan or Computed Tomography (CT) $[3,4]$. The MRI scan provides proper visualization of the anatomical structure of the tissues [5]. So, the MRI and CT is more suitable for disease diagnosis [6]. MRI uses magnetic field to diagnosis any changes occurring inside the brain and provide high quality results. CT scan uses radiations to check for abnormalities inside the tissues [7, 8]. Normally, brain tumor affects the Cerebral Spinal Fluid (CSF), it causes for strokes. The brain tumour cells are classified into two types such as Mass and Malignant $[9,10]$. Image segmentation and classification technique is used for brain tumors detection. The Medical Image Segmentation (MIS) scheme with few preferred properties used for segmentation process such as fast computation, accurate, minimum user interaction, and robust segmentation [11-13]. In diseases diagnosis, high resolution and non-invasive MR image have a significant control hence the image segmentation has more importance. The main objective of segmentation is dividing an input image into various regions or edges with similar properties. Several methods have been introduced to segment the image, many of them based on the basic two properties such as similarity based and discontinuity based. The success of medical image analysis attained accurate image segmentation algorithms, so 
an accurate segmentation of medical images is primary for clinical diagnosis and treatment planning [14]. In medical image processing, the classifier performance and length of selected feature subset are adopted as heuristic information [15]. This research uses the ARKFCM algorithm and auto encoder DNN classifier for brain tumor segmentation and classification. On the underlying stage, hybrid features are extracted on the segmented brain image by Gray-Level Co-occurrence Matrix (GLCM), Histogram of Oriented Gradient (HOG), and Local Binary Pattern (LBP) to improve the subset features. In next stage, the feature selection is done by KNN based GA to acquire the best feature values. Finally, meningioma tumor, Glioma tumor and pituitary tumors are classified by using auto encoder DNN classifier. Finally, the experimental outcome confirms that the proposed method delivers better classification performance compared to existing techniques.

This research paper is composed as follows, Section 2 presents an extensive survey of recent papers on brain tumour segmentation techniques. Section 3 briefly described an effective ARKFCMHFE-DNN methodology. Section 4 shows the comparative experimental result for the existing and ARKFCM-HFE-DNN method. The conclusion is made in section 5 .

\section{Literature survey}

The researchers have suggested a numbers of segmentation techniques for brain tumour detection in the medical field. A brief evaluation of a few significant segmentation techniques is presented in this section.

V. Jumb, M. Sohani, and A. Shrivas [16] proposed color image segmentation by implementing K-means clustering and Otsu's adaptive threshold technique. In this work, the Red Green Blue (RGB) images were converted into the HSV (Hue Saturation Value) color model and the value of channel was extracted. The Otsu's multi thresholding was applied to $\mathrm{V}$ channel to achieve better threshold value from the image. At the final stage, background subtraction was done with the morphological process. The proposed method was compared with 3-different kinds of segmentation techniques such as Fuzzy CMeans, region growing and Hill climbing with $\mathrm{K}$ means. This method improved by 57.79 of the PSNR value and 0.10 of the MSE. This paper does not consider any preprocessing, so this method may be affected by noise.

Md Sujan, N. Alam, S.A. Noman, and M.J. Islam [17] proposed a new method based on thresholding with morphological image analysis to find a brain tumor. Initially, the color image converted to grayscale and then noises was eliminated from the grayscale image by using filter technique. A grayscale image was converted to binary image, adding 0.3 threshold value with the Otsu's threshold value for accurately segment the tumor portion. Next, the morphological operation performed to find the brain tumor that consists of the brightest part of MRI. Accuracy $(84.72 \%)$ of the proposed method is not efficient in brain tumour detection because it does not use any type of classifier technique.

V. Anitha, and S. Murugavalli [18] presented an Adaptive Pillar K-Means (APKM) scheme used for successful segmentation. Classification methodology was done by two-tier classification approach. The two-tier classification method increased the accuracy value compared to the SVM classifier technique. In the proposed method, at the Self - Organizing Map Neural Network (SOMNN) trains the features extracted from the Discrete Wavelet Transform Blend wavelets (DWTBWs) and the resultant filter factors were trained by the $\mathrm{KNN}$ and the testing process was accomplished in two-stage. This method does not use hybrid feature extraction techniques to extract the tumor affected portion.

N. Abiwinanda, M. Hanif, S.T. Hesaputra, A. Handayani, and T. R. Mengko [19] proposed a brain tumour classification using CNN. They classified the brain tumours into three types: Glioma, Meningioma, and Pituitary by using CNN. They implemented the simplest possible architecture of the CNN (for example, one each of convolution, max pooling and flattening layers followed by a full connection from one hidden layer). The CNN was trained on a brain tumour dataset consisting of $3064 \mathrm{~T} 1$ weighted CEMRI images. Generally, the performance of the accuracy is very important in brain tumor detection. But, the accuracy of the proposed method is not evaluated.

J. Cheng, W. Huang, S. Cao, R. Yang, W. Yang, Z. Yun, Z. Wang, and Q. Feng [20] proposed a method to improve the performance of tumor classification. Initially, they used augmented tumor region via image dilation as the Region of Interest (ROI) instead of the original tumor portion because tumor surrounding tissue can also provide significant clues of a tumor type. In the second stage, augmented tumor portion split into a number of fine ring form sub portions. They evaluated the efficiency of this method based on a huge database with 3-feature extraction methods: Gray Level Co-occurrence Matrix (GLCM), intensity histogram and Bag of Words (BoW). But, SVM classifier does not achieve 


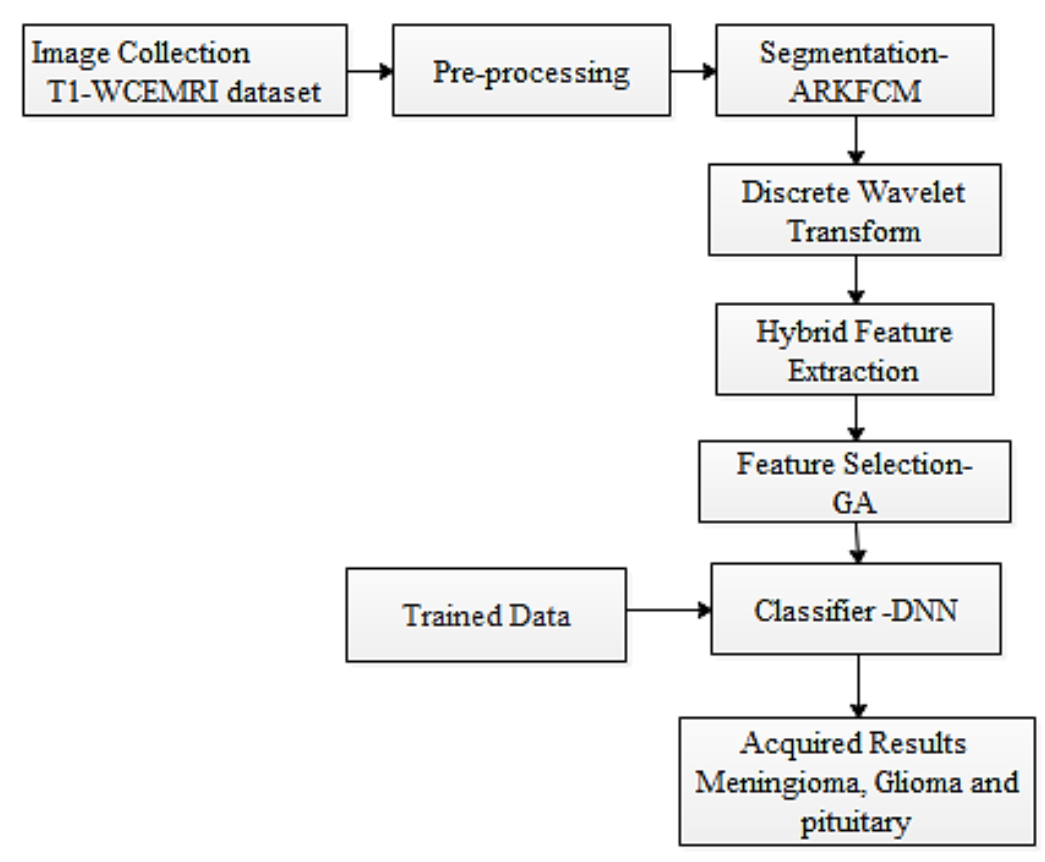

Figure.1 Block diagram of the ARKFCM-HFE-DNN method

the efficient accuracy because of the improper selection of the kernel.

The ARKFCM-HFE-DNN method is implemented to overcome the above-mentioned limitations and to improve the performance of the image segmentation process.

\section{ARKFCM-HFE- DNN methodology}

The major idea of the ARKFCM-HFE-DNN method is to increase system's accuracy in brain tumor detection by using ARKFCM techniques. The proposed brain tumor segmentation methodology has five steps, those are preprocessing, segmentation, feature extraction, feature selection, and classification. The proposed ARKFCM-HFE-DNN method's block diagram is represented in Fig. 1. A brief description of ARKFCM-HFE-DNN technique is determined below.

\subsection{Pre-processing and segmentation process}

In this research, the T1-W CEMRI dataset is used to compute the efficiency of the ARKFCM-HFEDNN method. Input images are resized like the $512 \times 512$. The pre-processed images are utilized to the segmentation process. The ARKFCM is undertaken for segmenting the images with and without tumor cell regions. This ARKFCM uses the heterogeneity of grayscales in neighborhood image for analyzing the local contextual information to replace the standard Euclidean distance with Gaussian radial basis kernel functions. Although, in these methods, some significant details in image may be lost because of the use of image smoothing operation and edge. Intially, this research compute the adaptive regularized parameter $\left(\varphi_{i}\right)$ connected with each pixel to control the contextual information using Eq. (1). The objective function is defined in Eq. (2).

$$
\varphi_{i}=\left\{\begin{array}{cc}
2+\omega_{i}, & \overline{x_{l}}<x_{i} \\
2-\omega_{i}, & \overline{x_{l}}>x_{i} \\
0, & \bar{x}_{\imath}=x_{i}
\end{array}\right.
$$

Where adaptive regularized parameter $\left(\varphi_{i}\right), \omega_{i}$ is, angular range and $x_{i} \& \bar{x}_{i}$ is mean gray scale.

$$
\begin{gathered}
J_{A R K F C M}=2\left[\sum_{i=1}^{N} \sum_{j=1}^{c} u_{i j}^{m}\left(1-K\left(x_{i}, v_{j}\right)\right)+\right. \\
\sum_{i=1}^{N} \sum_{j=1}^{c} \varphi_{i} u_{i j}^{m}\left(1-K\left(x_{i}, v_{j}\right)\right) \\
\sum_{j=1}^{c} u_{i j}=1, \quad u_{i j} \in[0,1], \quad 0 \leq \sum_{i=1}^{N} u_{i j} \leq N
\end{gathered}
$$

Where $u_{i j}^{m}$ is membership value, $x_{i} \& v_{j}$ are pixels, $m$ weighting exponent to degree of fuzziness and $K$ is kernel function.

Under the conditions specified in Eq. (3), the reduction of $J_{A R K F C M}(u, v)$ can be computed through an alternate optimization procedure using Eq. (4). 


$$
\begin{aligned}
& u_{i j}=\frac{\left(\left(1-K\left(x_{i}, v_{j}\right)\right)+\varphi_{i}\left(1-K\left(\bar{x}_{l}, v_{j}\right)\right)\right)^{-1 /(m-1)}}{\sum_{k=1}^{c}\left(\left(1-K\left(x_{i}, v_{j}\right)\right)+\varphi_{i}\left(1-K\left(\bar{x}_{l}, v_{k}\right)\right)\right)^{-1 /(m-1)}} \\
& v_{j}=\frac{\sum_{i=1}^{N} u_{i j}^{m}\left(K\left(x_{i}, v_{j}\right) x_{i}+\varphi_{i} K\left(\bar{x}_{l}, v_{j}\right) \bar{x}_{i}\right)}{\sum_{i=1}^{N} u_{i j}^{m}\left(K\left(x_{i}, v_{j}\right)+\varphi_{i} K\left(\bar{x}_{l}, v_{j}\right)\right)}
\end{aligned}
$$

When $\bar{x}$ is replaced with grayscale of average and median filter of original image, the algorithm is represented as $\mathrm{ARKFCM}_{1} / \mathrm{ARKFCM}_{2}$. When $\bar{x}_{l}$ is replaced with weighted image $\bar{\xi}_{i}$ defined in Eq. (6), the algorithm is represented as $A R K F C M_{\omega}$.

$$
\bar{\xi}_{i}=\frac{1}{2+\max \left(\varphi_{i}\right)}\left(x_{i}+\frac{1+\max \left(\varphi_{i}\right)}{N_{R}-1} \sum_{r \in N_{i}} x_{r}\right)
$$

Major steps for the proposed algorithms are as follows.

Step 1: Initialize threshold $\varepsilon=0.001, m=2$, loop counter $t=0, \mathrm{v}$ and $u^{(0)}$.

Step 2: Compute the adaptive regularization parameters $\varphi_{i}$.

Step 3: Compute $\bar{x}_{i}$ for $A R K F C M_{1}$ and $A R K F C M_{2}$.

Step 4: Compute cluster centers $v_{j}^{(t)}$ using $u^{(t)}$ as in equation (5).

Step 5: Compute the membership function $u^{(t+1)}$ with equation (4).

Step 6: If $\max \left\|u^{(t+1)}-u^{(t)}\right\|<\varepsilon$ or $t>100$ then stop; otherwise, update $t=t+1$ and go to step (4).

\subsubsection{Analyse wavelet coefficient in the segmented image using DWT}

To compute the wavelet coefficient of the segmented images, the DWT is used and it consider a square shaped function. The DWT technique has better compression energy and proper reconstruction with short support filters, low-computation, and no redundancy. The DWT follows the fuzzy de-noising procedure, which provides shift capable sub-bands and better directional selectivity with less redundancy. In the multi-resolution process, the real texture of the image is computed by zooming in and zooming out process. Usually, the images are decomposed into a number of sub-image at various resolution levels for preserving the low and highfrequency information. The DWT property helps to extract the information of the texture from the images. The square integral function $f(u)$ and wavelet transform $w$ is represented as the inner product $f$, and $\Psi(u)$ is original valued function. The wavelet function is given in the Eq. (7).

$$
w[f(s, \tau)]=\left(f, \Psi_{s, t}^{k}\right)=\int_{-\infty}^{\infty} f(u) \Psi_{s, t}^{k}(u) d u
$$

Where, $\quad \Psi_{s, t}^{k}(u)=\left(\frac{1}{\sqrt{s \Psi_{s, t}^{k}(u-\tau)}} / s\right) \quad$ denotes wave family, $s \in Z$ is scale, $\tau$ is translation and $k \in$ $\{h, v, d\}$ is orientation parameters. The orientation parameters $h, v$ and $d$ denote vertical, horizontal and diagonal direction respectively. The dyadic wavelet decomposition achieved during $s=2^{j}$ and $\tau=$ $2^{j}, n, j, n \varepsilon z$. The dyadic wavelet decomposition is a scalable sample of DWT; it follows a geometric sequence of ratio 2 . The following wavelet decomposition employs dyadic wavelets that are implemented using perfect reconstruction filter banks. By using wavelet function $\Psi(u)$ and the scaling function $\varphi(u)$, which showed in Eq. (8) and (9). The wavelet atoms describe by scaling and three mother atoms $\Psi^{h}, \Psi^{v}$ and $\Psi^{d}$. This mother atoms computed as the tensor products of 1-dimensional $\Psi(u)$ and $\varphi(u)$, that is denoted in Eq. (10) and (11).

$$
\begin{aligned}
& \Psi_{j, n}^{k}(u)=\frac{1}{\sqrt{2^{j}}} \Psi^{k}\left(\frac{u-2^{j} . n}{2^{j}}\right) \\
& \varphi_{j, n}^{k}(u)=\frac{1}{\sqrt{2^{j}}} \varphi^{k}\left(\frac{u-2^{j}, n}{2^{j}}\right) \\
& \varphi(u)=\varphi\left(u_{1}\right) \varphi\left(u_{2}\right), \Psi^{h}(u) \\
& \Psi^{v}(u)=\varphi\left(u_{1}\right) \Psi\left(u_{2}\right), \Psi^{d}(u)
\end{aligned}
$$

Two dimensional DWT is implemented by combination of down samplers and digital filter banks. The digital filter bank made up of a low-pass filter and high-pass filter. The number of the bank is grouped as per the desired functions in the wavelet configuration structure. Next, the rows and columns of the brain tumor images are separately undergone through the 1-dimensional wavelet transform in order to create 2-dimensional wavelet coefficient. The original brain tumor images $A_{2^{j+1}} f$ at resolution $2^{j+1}$ decompose into 4-subband images in the frequency domain. Three sub-band images such as $D_{2^{i}}^{h} f, D_{2^{i}}^{v} f, D_{2^{i}}^{d} f$ are the brain tumor images in resolution in vertical, horizontal and diagonal between the 4-subband images. The fourth image is an approximation image, $A_{2^{j}} f$ found at coarse resolution, so the entire brain tumor image $A_{2^{j+1}} f$ is denoted in the Eq. (12).

$$
A_{2^{j+1}} f=D_{2^{i}}^{h} f+D_{2^{i}}^{v} f+D_{2^{i}}^{d} f+A_{2^{j}} f
$$


The decomposed brain tumour sub-images are the 2-dimensional orthogonal wavelet. The results of the wavelet decomposition of an image is 4-orthogonal sub-bands such as Low-Low (LL) band, Low-High (LH) band, High-Low (HL) band and High-High $(\mathrm{HH})$ band, which is represented as $D_{2^{i}}^{h} f, D_{2^{i}}^{v} f, D_{2^{i}}^{d} f$ and $A_{2^{j}} f$ respectively.

\subsection{Hybrid feature extraction}

In ARKFCM-HFE-DNN method, the hybrid feature extraction performed on the transformed brain tumor image. The feature extraction is the action of mapping the image from image space to feature space. The GLCM is the well-known texture analysis technique, which computes the image features related to second order statistics of the input image and DWT process image. This research considered two-effective GLCM texture features: homogeneity and energy. The homogeneity measures the closeness of distribution features of the gray-level matrix. To quantitatively characterize the homogeneous texture portions for similarity, the Local Spatial Statistics (LSS) of the texture are computed using orientation and scale selective of Gabor filtering. Energy is calculated based on the number of repeated pairs and also the similarity is measured based on the normalized pixel pair distributions. This energy helps to reflect the depth and smoothness of the brain tumor image texture structure. In this research, the best feature is selected by using LBP descriptor. Here, the value of the central pixel is employed as a threshold. The normalized histogram can be computed and employed as the features. The histogram provides the information related to edges and spread of the discontinuities of the image. The LBP is most popular because it eases to calculate features and employed in many face recognition applications. Additionally, the Histogram of Oriented Gradients (HOG) descriptor is applied to the segmented image processing and computer version is used for extracting the optimal feature values. These feature values are given to the feature selection block as an input for selecting the best feature, which is described in the following section.

\subsection{Feature selection using KNN based GA}

The feature selection method selects the best features from the hybrid feature extraction. The classification involves consideration of the dataset before transferring the data to a classifier. It is recommended to consider only an important feature for selection. Hence, it is useful for choosing the significant and relevant features in this brain tumor detection. Furthermore, the feature selection is used during the classification to find the important feature that decreases the workload of the classifier and enhances the classification accuracy. In this research, KNN based GA minimizes the redundancy within input voxels and defines more relevance between input /output voxels. Then, the fitness function is calculated for input voxel subsets by using KNN that maximizes the mutual data between the voxel. Finally, the crossover \& mutation operators are utilized to detect the most active voxels by reducing the redundancy-based Fitness Function (FF). The GA selects the subset of features as the chromosomes and every chromosome is sent to the $\mathrm{KNN}$ for computing fitness value. The KNN employs each chromosome as a mask for capturing the features. The KNN defines a fitness value of each and every chromosome and GA utilizes these fitness values for the chromosome computation process. At the final stage, the GA finds an optimal subset of the feature.

\subsection{Classifier using auto encoder DNN}

The suspicious portions are classified by using auto encoder DNN classifier based on selected features. In this research, the proper selection of the auto encoder DNN will be a suitable solution for classification process when there is no prior knowledge about the distribution data. The auto encoder DNN typically works as feed forward networks and it is an unsupervised pre-training technique with greedy layer-wise training. In DNN, the data flow is achieved from the input layer to output layer without any looping function. The major advantage of auto encoder DNN classifier is the possibilities of lost value is very less.

The auto encoder is an encoding structure, it consists of neural network with multiple hidden layer, which shows in Fig. 2. Neurons of input layer denote the real input value. Every hidden layer can be seen as a higher level representation of previous layer, though it is generally non-trivial to define the exact meaning of every layer. The output layer is a sparse representation of input layer with similar dimensionality as the input.

Activation signals are interactively propagated forward through network utilizing Eq. (13). Until the output layer is reached. Neuron activation $a$ of every layer can be calculated by using Eq. (14).

$$
\left\{\begin{array}{c}
a_{(i)}^{(i)}=x^{(i)}, l=1 \\
a_{(i)}^{(i)}=\sigma\left(W^{l} a+b\right), l>1
\end{array}\right.
$$




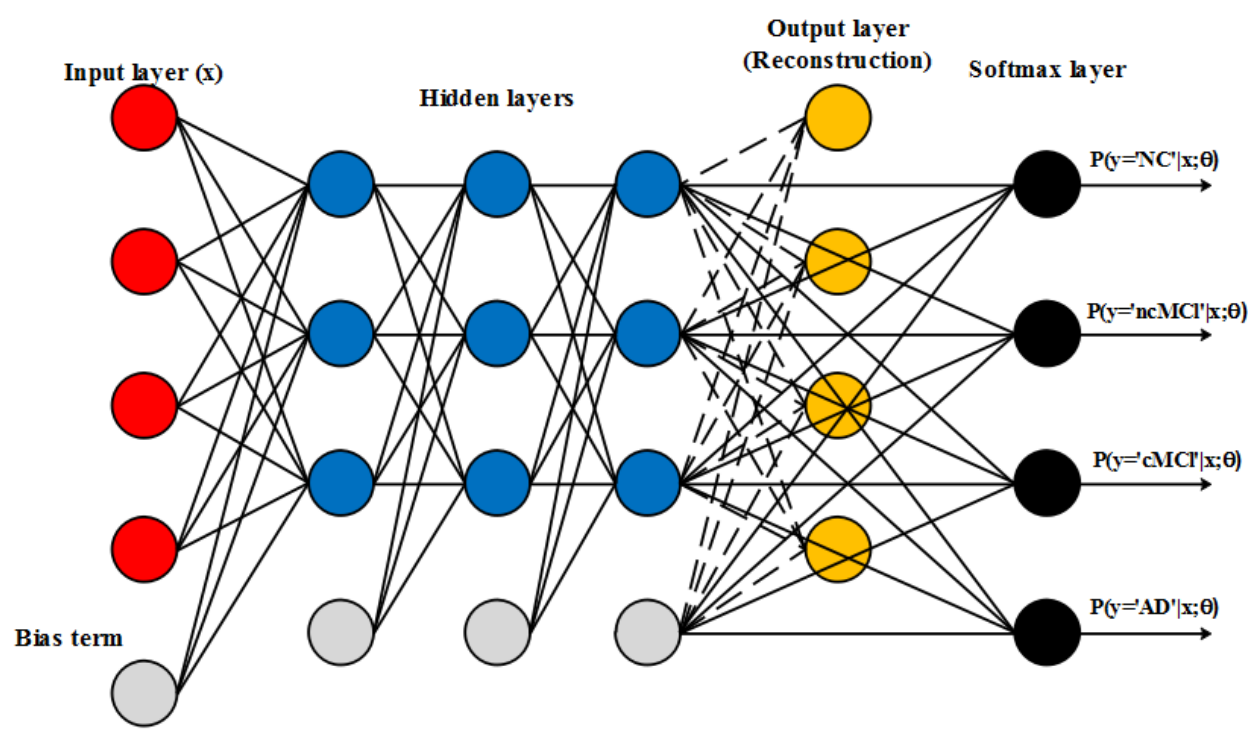

Figure.2 Structure of deep leaning

$$
h(W, b, x)=a^{(N)}
$$

Where $x$ represents unlabeled data $\left\{x^{(i)}\right\}_{i=1}^{m}, W$ represents weight matrix controls the activation effect between neurons on the neighbor layer, $b$ represents bias term, $\sigma$ represents activation function, it can be set to hyperbolic target function to introduce non-linearity for network to model complex relationship, and $h(W, b, x)$ represents input data as well as activation output layer. To train this unsupervised model, the representation loss is utilized as objective function for optimization as given in Eq. (15).

$$
\begin{aligned}
& L(W, b, x, z)=\min _{W, b} E(W, b, x, z)+\gamma\|W\|_{2}^{2}+ \\
& \beta K(W, b, x)
\end{aligned}
$$

Where, $\quad E(W, b, x, z)+\gamma\|W\|_{2}^{2} \quad$ denotes representation loss with squared error. Second term is weight decay that lead to small weights, third term is sparsity penalty regularized by $\beta$ with target activation of $\rho$ close to 0 , which enforces a sparse representation by penalizing the objective function utilizing Kullback Leibler divergence across $n$ training samples. It is defined in Eq. (16).

$$
K(W, b, x)=\sum_{j}^{n} I D_{K L}\left(\rho \| \sum_{i=1}^{m} h_{i}\left(x^{(i)} ; W, b\right)\right)
$$

This research trains the hidden layer of sparse auto-encoder one at time and stack them to form a complete $\mathrm{NN}$ by avoiding temporary output layer. A softmax output layer is added on top of trained auto encoder stack containing only previous hidden layer. Softmax layer employs a various activation function, which might have non-linearity, various from the one applied in previous layer. Softmax activation function is Eq. (17).

$$
h_{i}^{l}=\frac{e^{w_{i}^{l} h^{l-1+b_{i}^{l}}}}{\sum_{j} w_{i}^{l} h^{l-1+b_{i}^{l}}}
$$

Where $w_{i}^{l}$ is $i^{\text {th }}$ row of $W^{l}$ and $b_{i}^{l}$ is $i^{\text {th }}$ bias term of final layer. This research can employ $h_{i}^{l}$ as an estimator of $P(Y=i \mid x)$. Where $Y$ is the connected label of input data vector $x$. In this case, 4 output neurons at softmax layer can be interpreted as the probabilities of diagnosing an AD.

\section{Result and discussion}

The ARKFCM-HFE-DNN method was implemented in the MATLAB stimulator software version 2018b. The entire work implemented by using I3 system with 4 GB Random Access Memory (RAM). The performance criterion carried out by using some T1-weighted MRI scans. The T1weighted MRI scans were selected from the T1WCEMRI dataset. The performance of the ARKFCM-HFE-DNN method was implemented in terms of specificity, accuracy, sensitivity, and Fmeasure. To evaluate the classification efficiency of the ARKFCM-HFE-DNN method, the performance of the proposed method is compared with conventional methods: CNN and SVM on reputed dataset: T1-WCEMRI $[19,20]$.

\subsection{Performance measure}

The performance measurement is defined as a relationship between the input $\&$ output variables of 
the system by using suitable performance metrics like specificity and sensitivity. The general formula of specificity and sensitivity for brain tumour detection is given in the Eq. (18) and (19).

$$
\begin{aligned}
& \text { Sensitivity }=\frac{T P}{T P+T N} \times 100 \\
& \text { Specificity }=\frac{T N}{T N+T P} \times 100
\end{aligned}
$$

An accuracy and F-measure are the appropriate computation metrics for finding the effectiveness of the Meningioma, Glioma, and Pituitary in brain tumour detection. The performance measures are statistical variability and random errors. The general formula of accuracy given in the Eq. (20).

$$
\text { Accuracy }=\frac{T P+T N}{T P+T N+F P+F N} \times 100
$$

Irrelevant images can be determined by utilizing Precision, which is computed by using Eq. (21). Here, TP - True Positive, FP - False Positive, TN- True Negative and FP - False Negative.

$$
\text { Precision }=\frac{T P}{T P+F P}
$$

\subsection{T1-WCEMRI dataset}

From 2005 to 2010, the brain T1-weighed CEMRI dataset was acquired from Guangzhou, Nanfang Hospital, China, Tianjin Medical University, and General Hospital, China. This dataset composed of 3064 slices from 233 patients, containing 708meningiomas, 1426-Gliomas, and 930-pituitary tumours. The images have an enplane resolution of $512 \times 512$ with pixel size $0.49 \times 0.49 \mathrm{~mm}^{2}$. The slice thickness is $6 \mathrm{~mm}$ and the slice gap is $1 \mathrm{~mm}$. The tumour border was manually delineated by 3experienced radiologists. The T1-WCEMRI dataset contains three classes of brain images, those are Meningioma, Glioma and Pituitary tumour. Here, the performance evaluation validated for one sample image in each class. The validation result showed that the proposed method outperformed the existing methodologies by means of accuracy, sensitivity and specificity and precision.

\subsection{Performance analysis}

Performance evaluation of the three-stage brain tumours such as Glioma, Meningioma, and pituitary with feature selection represented in Table 1. In this research, the three-types of feature extraction techniques tested that are essentially analogous. Hybrid feature characterizes pairwise relations between 2-neighboring pixels and provides better results. Bow histogram uses image patch as a local feature, it considers the relations between several pixels. Therefore, the Bow histogram represented more discriminative and informative, yielding the best result.

Performance evaluation of the three-stage brain tumour with feature selection method is shown in Table 1. Performance analysis of the three-stage brain tumour without feature selection is shown in Table 2. In this research, after the segmentation, the texture feature and orientation gradient feature extracted from segmented images by using GLCM, LBP and HOG technique. Texture features were computed from the statistical distribution of the observed combination of intensities at a specified position relative to each image. After this process, GA based feature selection technique selected the best features from the hybrid feature extraction. The classification considered the dataset before transferring the data to a classifier. It is recommended to consider only an important feature for selection. Hence, it is useful for choosing the significant and relevant features in brain tumour detection than existing methods. After the feature selection process, the tumour portions were classified such as Glioma, Meningioma and Pituitary by using auto encoder DNN classifier based on selected features. The proposed method obtained better results compared to existing methods. In Table 1 , accuracy of the proposed method ARKFCM-HFEDNN with feature selection obtained $92.00 \%$, $99.32 \%$ and $99.12 \%$ of three different tumour classification. Here, the proposed method with feature selection classified the Meningioma, and pituitary tumour effectively than Glioma in terms of classification accuracy. In Table 2, accuracy of the proposed method ARKFCM-HFE-DNN without feature selection: obtained $83.24 \%, 84.32 \%$ and $83.55 \%$ of three different tumour classification.

Table 1. Performance evaluation of brain tumour classification of ARKFCM-HFE-DNN

\begin{tabular}{|c|c|c|c|c|}
\hline \multirow{2}{*}{ Tumour Stage } & \multicolumn{4}{|c|}{ Performance Analysis with feature selection } \\
\cline { 2 - 5 } & Accuracy \% & Sensitivity \% & Specificity \% & Precision \% \\
\hline Glioma & 92.00 & 92.11 & 100 & 100 \\
\hline Meningioma & 99.32 & 100 & 100 & 100 \\
\hline Pituitary & 99.12 & 100 & 96.01 & 93.21 \\
\hline
\end{tabular}




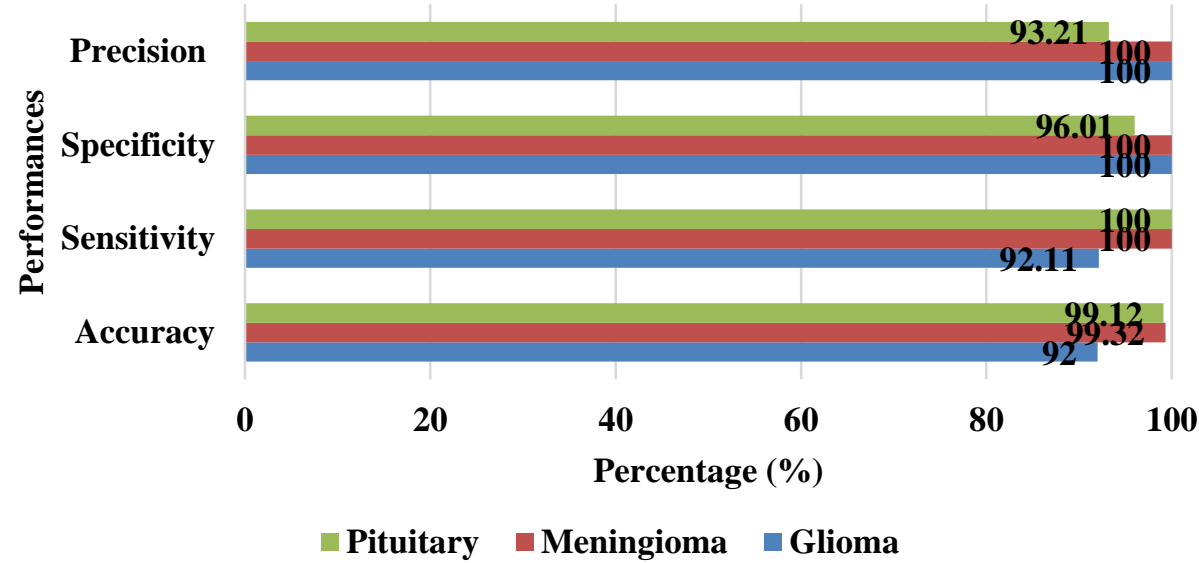

Figure.3 Comparison analysis of different brain tumour regions with feature selection of ARKFCM-HFE-DNN method

Table 2. Performance analysis of brain tumour classification without feature selection

\begin{tabular}{|c|c|c|c|c|}
\hline \multirow{2}{*}{ Tumour Stage } & \multicolumn{4}{|c|}{ Performance Analysis without feature selection } \\
\cline { 2 - 5 } & Accuracy \% & Sensitivity \% & Specificity \% & Precision \% \\
\hline Glioma & 83.24 & 72.25 & 94.65 & 86.35 \\
\hline Meningioma & 84.34 & 76.38 & 86.25 & 73.89 \\
\hline Pituitary & 83.55 & 100 & 94.65 & 89.35 \\
\hline
\end{tabular}

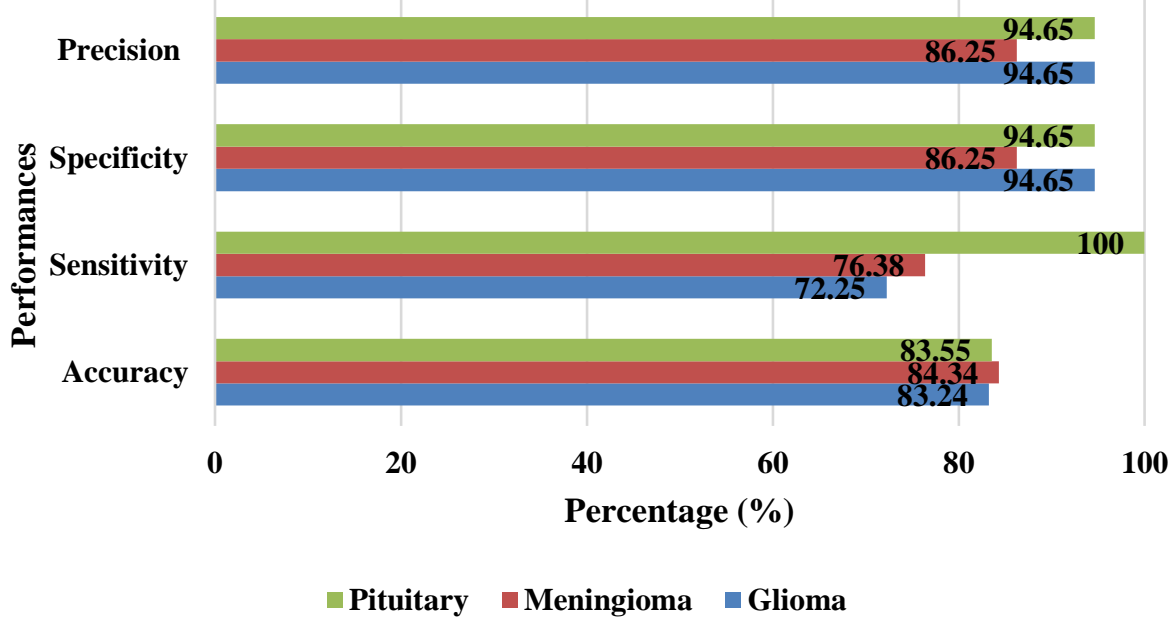

Figure.4 Comparison performance of different brain tumour regions without feature selection of ARKFCM-HFE-DNN method

Proposed method with feature selection method delivered better classification accuracy compared to without feature selection. Figs. 3 and 4 shows the graphical comparison of accuracy, sensitivity, specificity and precision for abnormal brain tumour classification with feature selection and without feature selection.

In this section, performance comparison of the existing and ARKFCM-HFE-DNN method are shown in Table 3. In Abiwinanda [19], CNN based tumour classification method was obtained $84.16 \%$ of accuracy. J. Cheng [20], Support Vector Machine (SVM) based abnormal tumour classification method was obtained $91.14 \%$ of accuracy. In Table 3, the accuracy of proposed ARKFCM method is obtained efficient classification accuracy compared to Abiwinanda [19] and J. Cheng [20]. The proposed ARKFCM-HFE-DNN method consist hybrid feature extraction, feature selection and classification experiment, were carried out on T1-WCEMRI database for validating the results in terms of 
Table 3. Performance comparison of the existing and ARKFCM-HFE-DNN method

\begin{tabular}{|c|c|c|c|}
\hline Authors & Database & Classification Scheme & Tumour Classification Accuracy (\%) \\
\hline Abiwinanda [19] & T1-W CEMRI & Convolutional Neural Network & 84.16 \\
\hline J. Cheng [20] & T1-W CEMRI & Support Vector Machine & $91.14 \%$ \\
\hline Proposed method & T1-WCEMRI & Auto encoder DNN & $97.33 \%$ \\
\hline
\end{tabular}
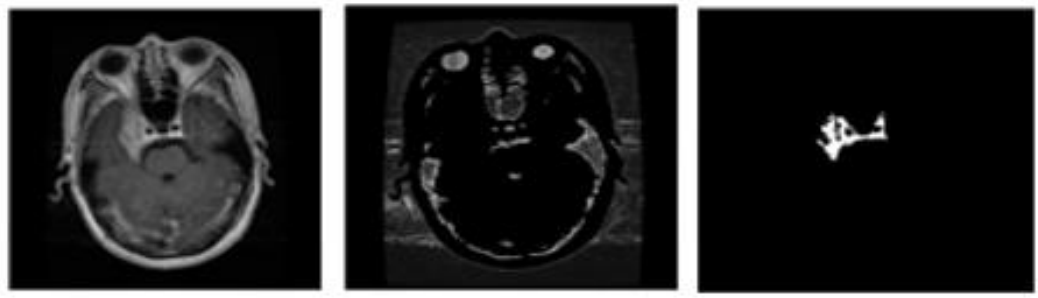

Figure.5 Input, cluster and segmented image of Glioma brain tumour image
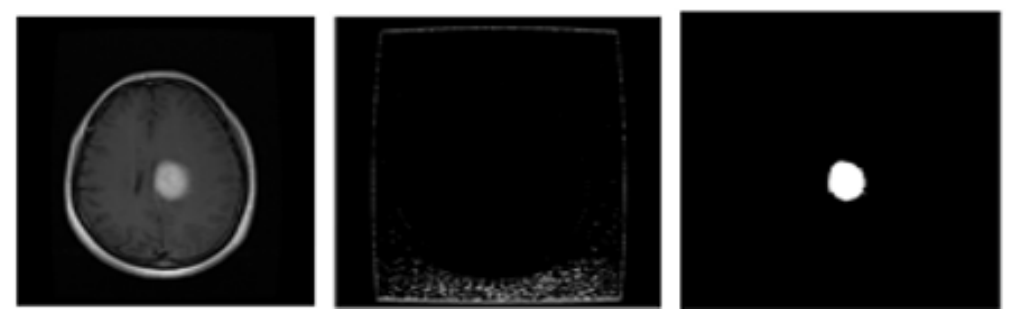

Figure.6 Input, cluster and segmented image of Meningioma brain tumour
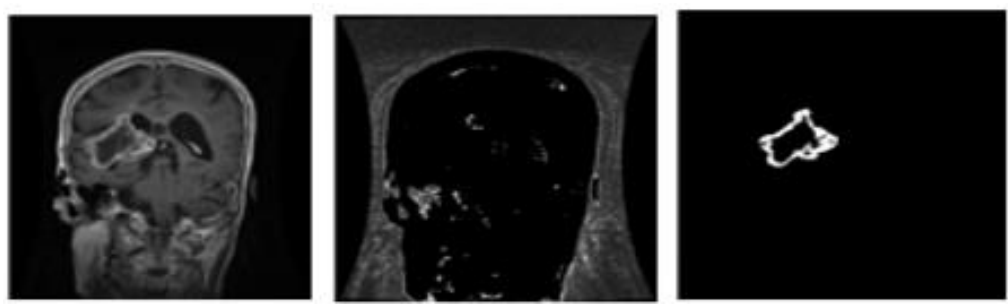

Figure.7 Input, cluster and segmented image of Pituitary brain tumour image

accuracy. The proposed method achieved $97.33 \%$ of accuracy which is higher than accuracy of existing brain tumour detection methods. Figs. 5, 6 and 7 depicts the input, cluster and segmented image of three brain tumour.

The MRI brain image segmentation was performed using ARKFCM. In order to find the classification accuracy, the various texture and gradient orientation features extracted by using GLCM, HOG, and LBP techniques. The DNN is utilized to classify the abnormal brain image into Meningioma, Glioma and Pituitary. It can be concluded from the experimental results that the proposed ARKFCM-HFE-DNN method is more suitable for abnormal brain tumour detection and classification compared to existing methods in terms of accuracy.

\section{Conclusion}

Image segmentation process plays an important role in medical image processing. In this work, the
ARKFCM segmentation technique was employed to segment the brain tumour portion of the brain image. The HFEs (GLCM, HOG, and LBP) extracted the optimal feature values from segmented region. Then, the feature selection method was applied to the feature extraction data for best feature selection. Finally, the suspicious portions were classified by using auto encoder DNN classifier based on selected features. The ARKFCM-HFE-DNN method delivered better performance by means of accuracy $6.19 \%$ compared to the existing confusion matrixGLCM method. The accuracy rate is $97.33 \%$, which is much promising to identify the abnormal brain tumours classification from patient MRI image, and the proposed method can be used to classify the various types of tumour according through medical diagnosis system. In future work, the hybrid feature extraction with an optimized algorithm can be implemented to further improve the brain tumour detection in terms of accuracy, sensitivity, specificity and so on. 


\section{References}

[1] A. Norouzi, M.S.M. Rahim, A. Altameem, T. Saba, A.E. Rad, A. Rehman, and M. Uddin, "Medical image segmentation methods, algorithms, and applications", IETE Technical Review, Vol.31, No. 3, pp.199-213, 2014.

[2] R. Al-Awadi, M. Elmogy, and E. AbdelMaksoud, "Brain tumour segmentation based on a hybrid clustering technique", Egyptian Informatics Journal, Vol.16, No.1, pp.71-81, 2015.

[3] J. Patel and K. Doshi, "A study of segmentation methods for detection of tumour in brain MRI", Advance in Electronic and Electric Engineering, Vol.4, No.3, pp.279-284, 2014.

[4] P.K. Mallick, B.S. Satapathy, M.N. Mohanty, and S.S. Kumar, "Intelligent technique for CT brain image segmentation", In: Proc. of the 2nd International Conference on Electronics and Communication Systems, pp.1269-1277, 2015.

[5] D. Selvaraj and R. Dhanasekaran, "Mri brain image segmentation techniques-A review", Indian Journal of Computer Science and Engineering, Vol.4, No.5, pp.364-381, 2013.

[6] D.P. Malashree and B.A. Thomas, "Brain Tumour Detection by MRI and Ct Scan Images", International Journal of Current Trends in Engineering \& Research, Vol.2, No.6, pp.550555, 2016.

[7] I. Ahmed, Q. Nida-Ur-Rehman, G. Masood, and M. Nawaz, "Analysis of Brain MRI for Tumour Detection \& Segmentation", In: Proc. of the World Congress on Engineering, Vol.1, p.25, 2016.

[8] K.B. Vaishnavee and K. Amshakala, "An automated MRI brain image segmentation and tumour detection using SOM-clustering and Proximal Support Vector Machine classifier", In: Proc. of International Conference on Engineering and Technology, pp.1-6, 2015.

[9] R.S. Kabade and M.S. Gaikwad, "Segmentation of Brain Tumour and Its Area Calculation in Brain MR Images using K-Mean Clustering and Fuzzy CMean Algorithm", International Journal of Computer Science \& Engineering Technology, Vol.4 No.05, pp.524-531, 2013.

[10] R.P. Joseph, C.S. Singh, and M. Manikandan, "Brain tumour MRI image segmentation and detection in image processing", International Journal of Research in Engineering and Technology, Vol.3, No.1, pp.1-5, 2014.

[11] H.A. Aslam, T. Ramashri, and M.I.A. Ahsan, "A new approach to image segmentation for brain tumour detection using pillar K-means algorithm", International Journal of Advanced Research in Computer and Communication Engineering, Vol.2, No.3, pp.1429-1436, 2013.

[12] M. Angulakshmi and G.G.L. Priya, "Automated brain tumour segmentation techniques-A review", International Journal of Imaging Systems and Technology, Vol.27, No.1, pp.6677, 2017.

[13] M. Banerjee, R. Chowdhury, and S.K. Bandyopadhyay, "Detection of Brain Tumour from MRI of Brain", International Journal of Information Research and Review, Vol.02, No. 12, pp.1555-1559, 2015.

[14] B. Wilson and J.P.M. Dhas, "An experimental analysis of Fuzzy C-means and K-means segmentation algorithm for iron detection in brain SWI using Matlab", International Journal of Computer Applications, Vol.104, No.15, pp.36-38, 2014.

[15] C. Cernazanu-Glavan and S. Holban, "Segmentation of bone structure in X-ray images using convolutional neural network", Adv. Electr. Comput. Eng, Vol.13, No.1, pp.8794, 2013.

[16] V. Jumb, M. Sohani, and A. Shrivas, "Color image segmentation using K-means clustering and Otsu's adaptive thresholding", International Journal of Innovative Technology and Exploring Engineering, Vol.3, No.9, pp.7276, 2014.

[17] Md Sujan, N. Alam, S.A. Noman, and M.J. Islam, "A Segmentation based Automated System for Brain Tumour Detection", International Journal of Computer Applications, Vol.153, No.10, pp.41-49, 2016.

[18] V. Anitha and S. Murugavalli, "Brain tumour classification using two-tier classifier with adaptive segmentation technique", IET Computer Vision, Vol.10, No.1, pp.9-17, 2016.

[19] N. Abiwinanda, M. Hanif, S.T. Hesaputra, A. Handayani, and T. R. Mengko, "Brain Tumour Classification Using Convolutional Neural Network", In: Proc. of World Congress on Medical Physics and Biomedical Engineering, pp. 183-189, 2019.

[20] J. Cheng, W. Huang, S. Cao, R. Yang, W. Yang, Z. Yun, Z. Wang, and Q. Feng, "Correction: Enhanced Performance of Brain Tumour Classification via Tumour Region Augmentation and Partition", PloS one, Vol.10, No.12, pp.e0144479, 2015. 